\title{
Embedded Correlated Wavefunction Schemes: Theory and Applications
}

\author{
Florian Libisch, ${ }^{\dagger}$ Chen Huang, ${ }^{\ddagger}$ and Emily A. Carter*, ${ }^{*}$ \\ ${ }^{\dagger}$ Institute for Theoretical Physics, Vienna University of Technology, 1040 Vienna, Austria \\ ॠTheoretical Division, Los Alamos National Laboratory, Los Alamos, New Mexico 87545, United States \\ ${ }^{\S}$ Department of Mechanical and Aerospace Engineering, Program in Applied and Computational Mathematics, and Andlinger Center \\ for Energy and the Environment, Princeton University, Princeton, New Jersey 08544, United States
}

CONSPECTUS: Ab initio modeling of matter has become a pillar of chemical research: with ever-increasing computational power, simulations can be used to accurately predict, for example, chemical reaction rates, electronic and mechanical properties of materials, and dynamical properties of liquids. Many competing quantum mechanical methods have been developed over the years that vary in computational cost, accuracy, and scalability: density functional theory (DFT), the workhorse of solid-state electronic structure calculations, features a good compromise between accuracy and speed. However, approximate exchange-correlation functionals limit DFT's ability to treat certain phenomena or states of matter, such as charge-transfer processes or strongly correlated materials. Furthermore, conventional DFT is purely a ground-state theory: electronic excitations are beyond its scope. Excitations in molecules are routinely calculated using time-dependent DFT linear response; however applications to condensed matter are still limited.

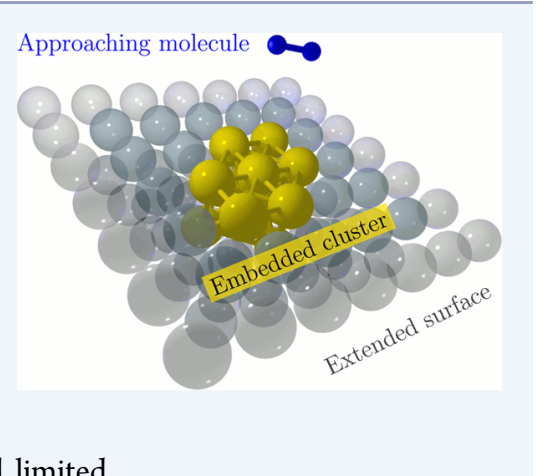
By contrast, many-electron wavefunction methods aim for a very accurate treatment of electronic exchange and correlation. Unfortunately, the associated computational cost renders treatment of more than a handful of heavy atoms challenging. On the other side of the accuracy spectrum, parametrized approaches like tight-binding can treat millions of atoms. In view of the different (dis-)advantages of each method, the simulation of complex systems seems to force a compromise: one is limited to the most accurate method that can still handle the problem size. For many interesting problems, however, compromise proves insufficient. A possible solution is to break up the system into manageable subsystems that may be treated by different computational methods. The interaction between subsystems may be handled by an embedding formalism.

In this Account, we review embedded correlated wavefunction (CW) approaches and some applications. We first discuss our density functional embedding theory, which is formally exact. We show how to determine the embedding potential, which replaces the interaction between subsystems, at the DFT level. CW calculations are performed using a fixed embedding potential, that is, a non-self-consistent embedding scheme. We demonstrate this embedding theory for two challenging electron transfer phenomena: (1) initial oxidation of an aluminum surface and (2) hot-electron-mediated dissociation of hydrogen molecules on a gold surface. In both cases, the interaction between gas molecules and metal surfaces were treated by sophisticated CW techniques, with the remainder of the extended metal surface being treated by DFT. Our embedding approach overcomes the limitations of conventional Kohn-Sham DFT in describing charge transfer, multiconfigurational character, and excited states. From these embedding simulations, we gained important insights into fundamental processes that are crucial aspects of fuel cell catalysis (i.e., $\mathrm{O}_{2}$ reduction at metal surfaces) and plasmon-mediated photocatalysis by metal nanoparticles. Moreover, our findings agree very well with experimental observations, while offering new views into the chemistry. We finally discuss our recently formulated potential-functional embedding theory that provides a seamless, first-principles way to include back-action onto the environment from the embedded region.

\section{INTRODUCTION}

A system of (nonrelativistic) interacting electrons is governed by the many-body Schrödinger equation. Within the approximation of a finite basis set, a full configuration interaction (CI) expansion that includes all possible electron configurations yields exact results. However, full CI scales factorially with the number of particles $N$. Truncated correlated wavefunction (CW) methods, which scale polynomially with $N$, offer the most accurate practical solution of the Schrödinger equation. For example, coupled cluster single and double excitations with perturbative triples, $\operatorname{CCSD}(\mathrm{T}){ }^{1}{ }^{1}$ scales as $N^{7}$. While this scaling is better than factorial, still only molecules of moderate size can be treated, unless specially designed, reduced-scaling algorithms are employed. ${ }^{2-4}$ Extending these methods to condensed matter has been much more challenging and is prohibitively expensive. $^{5-7}$

Special Issue: Beyond QM/MM: Fragment Quantum Mechanical Methods

Received: February 28, 2014

Published: May 30, 2014 
As a less demanding alternative, Kohn-Sham density functional theory (KS-DFT) enjoys wide popularity as the method of choice for electronic structure calculations because it scales as $\mathrm{N}^{3}$ or less. KS-DFT rests on the Hohenberg-Kohn theorems, which prove a one-to-one mapping between the ground-state, $3 \mathrm{~N}$-dimensional, many-electron wavefunction of interacting electrons, $\Psi$, and the corresponding ground-state density, $\rho_{\mathrm{G}}(r)$, a three-dimensional object. Although the Hohenberg-Kohn theorems guarantee this mapping, they offer no constructive way to determine it. The unknown physics lies in the so-called exchange-correlation (XC) potential. While many approximations to the latter have been proposed, its exact form remains elusive. Nevertheless, DFT applications on $\sim 100$ atoms are now routine.

One strategy to deal with large, complex systems requiring high accuracy is to treat a small subset of atoms with expensive CW methods embedded in an extended system described by a more cost-effective technique. Several embedding schemes exist. ${ }^{8}$ Electrostatic embedding is used for ionic crystals, where the environment is well approximated by point charges. If environment polarization is important, shell models can be employed. ${ }^{9}$ Quantum mechanics/molecular mechanics (QM/ $\mathrm{MM}$ ) is employed to study covalently bonded systems such as semiconductor surfaces or biomolecules, as well as solutes in solution. In $\mathrm{QM} / \mathrm{MM}$, the embedded region is treated using quantum mechanics, while the background region is described by classical force fields. ${ }^{10}$ The related ONIOM embedding approach introduces several layers, each of which may be treated by increasingly sophisticated ab initio QM methods. ${ }^{11}$ In projection-based schemes, ${ }^{12}$ the kinetic energy of the entire system is treated exactly, and a DFT description of the outer parts of a molecule may be combined with correlated wavefunction methods for an embedded region, to treat, for example, transition metal centers embedded in large molecules. ${ }^{13}$ It is not clear how to extend this projection approach beyond molecules to condensed matter. Another promising approach is density-matrix embedding, in which the local density matrix of a low-level method like a DFT approximation in the region of interest is matched to the results of a high-level method by adjusting a nonlocal embedding potential. ${ }^{14}$

Metals require different strategies, and multiple embedding schemes exist. In Green's function embedding, ${ }^{15}$ one defines an artificial boundary to separate the region of interest and the environment. Then the environment is replaced by the surface Green's function on the boundary of the region of interest. However, it is not clear how to solve for the region of interest with methods beyond mean-field theories. Another effective means of metallic embedding, density functional embedding theory (DFET), was first proposed by Govind, Wang, and Carter, inspired by the work of Cortona, ${ }^{16}$ and Wesolowski and Warshel. ${ }^{17}$ In DFET, the total electron density is partitioned into the electron density of a cluster of atoms of interest plus the electron density of the environment. Both the cluster and the environment are treated quantum mechanically. The influence of the environment on the cluster is replaced by an embedding potential. ${ }^{8}$

DFET combines a periodic DFT description of the environment with a CW treatment of the cluster. This approach has been used to study, for example, ground and excited states of $\mathrm{CO}$ molecules on palladium and copper surfaces $^{18-23}$ and strongly correlated Kondo states of cobalt on copper and silver surfaces. ${ }^{24-28}$ The embedding potential is formulated as density functional derivatives of a formally exact (within DFT) interaction energy linking the cluster and the environment. ${ }^{8}$ The original formulation required using approximate kinetic energy density functionals (KEDFs) to evaluate the kinetic energy component of the interaction between subsystems. Since the exact form of the KEDF is unknown, the reliability of embedding results could not be guaranteed.

In this Account, we review two recent advances in DFET: (i) a new DFET that delivers an ab initio embedding potential and eliminates the need for approximate $\mathrm{KEDFs}^{29}$ and (ii) a reformulation of DFET as a potential-functional embedding theory $(\mathrm{PFET})^{30}$ that provides a self-consistent way to couple different subsystems in a seamless, first-principles manner. While numerically more demanding, PFET self-consistently accounts for the back-action of the embedded cluster on the environment, or in the case of more than two subsystems, for the interaction of each subsystem with all the others. Recent DFET applications are discussed, including charge-transferinduced dissociation of an oxygen molecule on an aluminum surface $^{31}$ and hot-electron-induced dissociation of hydrogen molecules on gold nanoparticles. ${ }^{32,33}$ We close with a brief summary and outlook on future development.

\section{DENSITY-FUNCTIONAL EMBEDDING THEORY}

In DFET, we partition the total system into subsystems, by grouping atoms into subgroups. In the simplest case, the system is decomposed into two subsystems, a cluster and an extended environment [Figure 1a]. Usually, the cluster is defined by a

(a)

a) total system

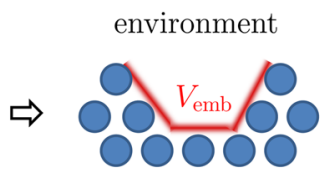

cluster

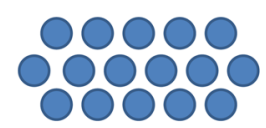

(b)
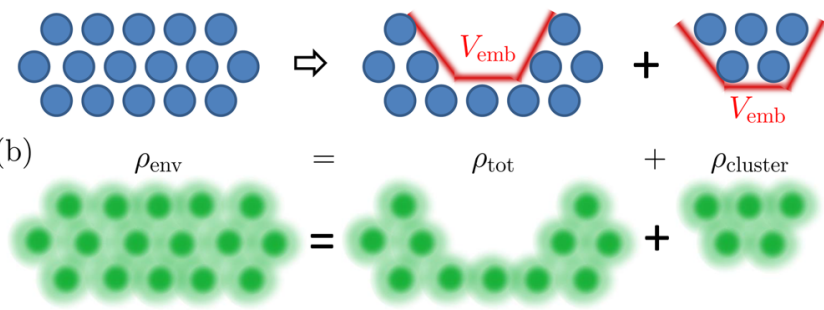

Figure 1. (a) Schematic view of density-functional embedding theory: a bulk system is partitioned into a (possibly periodic) bulk environment and a finite-sized cluster. Their interaction is mediated by an embedding potential, $V_{\text {emb }}$ (red). (b) The embedding potential is chosen such that the sum of the electron densities of cluster, $\rho_{\text {cluster, }}$, and environment, $\rho_{\text {env }}$, reproduces the total electron density, $\rho_{\text {tot }}$ which is calculated in advance.

specific set of atoms comprising the region of interest. The other atoms are considered as the environment. The total energy of the entire system is then decomposed as

$$
E_{\text {tot }}=E_{\text {cluster }}\left[\rho_{\text {cluster }}\right]+E_{\text {env }}\left[\rho_{\text {env }}\right]+E_{\text {int }}\left[\rho_{\text {cluster }}, \rho_{\text {env }}\right]
$$

where we have introduced the energy of the isolated cluster $\left(E_{\text {cluster }}\right)$ and isolated environment $\left(E_{\text {env }}\right)$, and the interaction energy, $E_{\text {int }}$ that describes all additional energy contributions due to their interaction.

The embedding potential for the cluster (environment) due to the environment (cluster) is the functional derivative of the interaction energy with respect to the cluster (environment) electron density, 


$$
\frac{\delta E_{\text {int }}}{\delta \rho_{\text {cluster }}}=V_{\text {emb }}^{\text {cluster }}, \quad \frac{\delta E_{\text {int }}}{\delta \rho_{\text {env }}}=V_{\mathrm{emb}}^{\mathrm{env}}
$$

This definition allows for an arbitrary partitioning of the total electron density, as long as the sum of the cluster and environment electron densities matches the electron density of the total system. ${ }^{29}$ We enforced a novel constraint in which the two embedding potentials are required to be equivalent. Such a constraint yields a unique, global embedding potential, that is, $V_{\mathrm{emb}}^{\text {cluster }}=V_{\mathrm{emb}}^{\mathrm{env}}=V_{\mathrm{emb}}$, resulting in a unique electron density partitioning. ${ }^{29}$ Such a unique density partitioning was first shown for the chemical reactivity potential, ${ }^{34,35}$ and also naturally appears in the (formally exact) partition DFT of Cohen and Wasserman, ${ }^{36}$ where the total system is decomposed into subsystems each handled by DFT. The goal in DFET is to solve for this unique embedding potential, $V_{\mathrm{emb}}$, which then is used as an external potential in more refined quantum mechanics calculations on the embedded cluster. To obtain $V_{\text {emb }}$, we first calculate the total electron density $\rho_{\text {tot }}$. In principle, any electronic structure method could be used for this task; here we employ KS-DFT to solve for $\rho_{\text {tot }}$. The subsystems are then solved individually using KS-DFT with the same XC functional, with the current iteration's $V_{\text {emb }}$ as an additional external potential. In practice, we use the same bulk supercell for the total system and the individual subsystem calculations. We aim to find a $V_{\text {emb }}$ such that the sum of subsystem electron densities matches the total electron density [Figure $1 \mathrm{~b}$ ]. Such a $V_{\mathrm{emb}}$ can be efficiently solved for by an optimized-effective potential procedure. ${ }^{29}$ The accuracy of the embedding potential is then determined by the level of XC functional used. In all of our applications thus far, $V_{\mathrm{emb}}$ is derived from KS-DFT, since it is often sufficient to treat the environment and the interaction between the environment and cluster at the DFT level. By contrast, the cluster often requires treatment by quantum mechanics methods beyond DFT.

Once $V_{\text {emb }}$ is obtained, one proceeds with the embedded CW calculations on the cluster with $V_{\text {emb }}$ as an external potential. CW techniques generally expand the wavefunction in a Gaussian basis set. After determining the required matrix elements of the many-particle Hamiltonian, $\mathcal{H}$, all calculations can be compactly written in matrix form. We represent $V_{\text {emb }}(r)$ on a real-space grid, replicated periodically around the entire cluster. For embedded CW calculations, the required substitution $\mathcal{H}_{\text {emb }}=\mathcal{H}+V_{\text {emb }}$ consequently only enters the setup of the calculations, where all necessary matrix elements are calculated. This offers the substantial advantage that most state-of-the-art quantum chemistry packages can be used with minimal modifications, which only pertain to the calculation of matrix elements.

A locally corrected total energy (beyond DFT) can be obtained according to first order perturbation theory, which yields a surprisingly simple and accurate expression in terms of the DFT $\left(E_{\mathrm{emb}, \mathrm{DF}}^{\mathrm{DFT}}\right)$ and $\mathrm{CW}\left(E_{\mathrm{emb}, \mathrm{cl}}^{\mathrm{CW}}\right)$ energies of the embedded cluster, ${ }^{29}$

$$
E_{\text {tot }}=E_{\text {tot }}^{\mathrm{DFT}}+\left(E_{\mathrm{emb}, \mathrm{cluster}}^{\mathrm{CW}}-E_{\mathrm{emb}, \mathrm{cluster}}^{\mathrm{DFT}}\right)
$$

The terms in parentheses can be interpreted as a correction to the DFT energy of the entire system, $E_{\text {tot }}^{\mathrm{DFT}}$. This correction accounts for the exchange-correlation energy of the electrons in the cluster that is not accounted for properly by DFT. The subtraction in eq 3 minimizes errors due to the relatively small size of typical Gaussian basis sets: we use the same basis set to evaluate the DFT and CW contributions to the embedded cluster.

Note the formal similarity of our energy expression in eq 3 with the ONIOM approach; in the latter, the correction on the cluster is assembled in a very similar way, but no embedding potential is used in the calculation. ${ }^{11}$ While such a simpler approach works well for covalently bonded systems, it fails to account for the mutual polarization of the cluster and its environment. Such a situation is unavoidable in metals, where the electron density distribution features delocalized Bloch states dramatically different from a small metal cluster. Our embedding potential thus helps to restore the proper electronic structure in such an embedded metal cluster. Reliable embedded CW calculations can then be performed, as discussed in the following section.

\section{APPLICATIONS}

\subsection{Initial Oxidation of an Aluminum Surface}

The preceding DFET formalism has thus far been used primarily to investigate molecules interacting with metal surfaces, a ubiquitous phenomenon central to understanding catalysis, corrosion, and more. Unfortunately, details of the processes involved remain poorly understood. ${ }^{37,38}$ Consider, for example, the seemingly simple event of dissociative adsorption of an oxygen molecule on a clean aluminum surface. This case nicely illustrates the complexity in elucidating surface reaction mechanisms from theory: experiments consistently find greatly reduced sticking probabilities at low incident energies ${ }^{39,40}$ suggestive of the presence of a barrier to dissociation. By contrast, straightforward KS-DFT calculations show no such barrier. ${ }^{41}$ On one hand, explanations in terms of spin selection rules have been put forward. ${ }^{42,43}$ On the other hand, CW calculations on isolated aluminum clusters obtaining a barrier suggest approximate $\mathrm{XC}$ functionals as the culprit. ${ }^{44}$ However, the barrier might be due to finite cluster sizes used. Thus, the origin of the adsorption barrier was still unclear.

To apply our embedding approach to this problem, we first carve out a cluster [typically containing a dozen aluminum atoms, see, for example, Figure 2a] from a clean aluminum surface without the $\mathrm{O}_{2}$ molecule. We use the discussed DFET embedding algorithm to calculate the embedding potential for this aluminum cluster [see Figure $2 b, c]$. The embedding potential forms an attractive shell at the boundary of the cluster toward the rest of the extended surface, simulating the metallic bonding between the cluster and the rest of the bulk material [see Figure 2c,d]. By performing embedded CW and DFT calculations using this fixed embedding potential, we evaluate the locally corrected energy of eq 3 at different positions and bond lengths of the approaching $\mathrm{O}_{2}$ molecule. ${ }^{31}$ The embedding potential is not updated for each position of the approaching $\mathrm{O}_{2}$. The reason is threefold: From a numerical perspective, it is too costly to recalculate $V_{\mathrm{emb}}$ for each of the approximately 200 points required for a single two-dimensional potential energy surface. From a modeling perspective, the short screening length of metal nanoclusters shields the effect of the approaching $\mathrm{O}_{2}$ from the remainder of the metal surface, as we have verified numerically. ${ }^{31}$ Most importantly, from a physics perspective, the adsorption involves charge transfer from the surface to the molecule. Since DFT does not reliably model charge transfer, ${ }^{45}$ an embedding potential based on DFT would carry over the inaccuracy of DFT to the boundary 
(a)

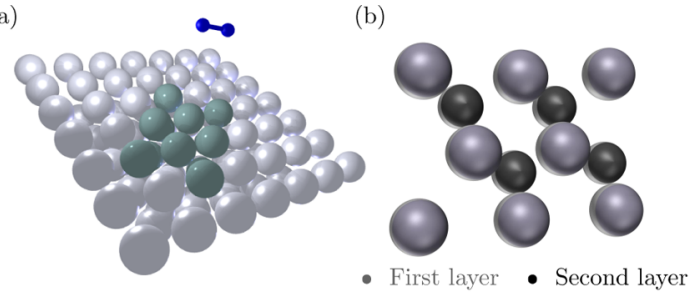

(c)

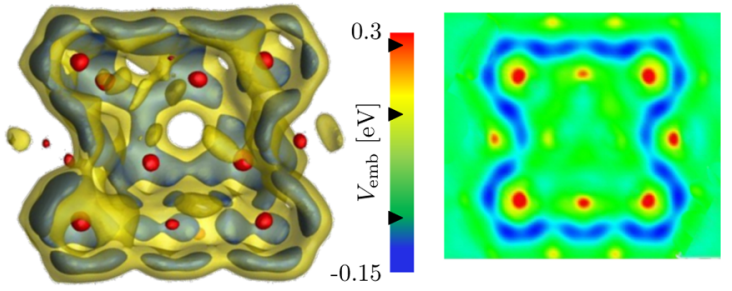

Figure 2. (a) Aluminum(111) surface and approaching oxygen molecule (blue). A possible selection of atoms for a bridge-site cluster is shaded. (b) Bridge-site aluminum cluster [see shaded atoms in panel a] consisting of 12 atoms (eight in the first and four in the second layer). (c) Equipotential surface plot of the embedding potential for the bridge cluster in panel b. Black triangles in the color bar mark the contour values drawn. (d) $2 \mathrm{D}$ cut of the embedding potential shown in c along the first atomic layer.

conditions of the embedded CW calculations, defeating the purpose of the entire embedding calculation.

In contrast to KS-DFT, our embedded CW theory yields a finite barrier toward dissociation [compare Figure 3a,b], for different absorption sites and parallel as well as perpendicular $\mathrm{O}_{2}$ orientations. ${ }^{31}$ The average barrier height of $300-600 \mathrm{meV}$ is consistent with experimental measurements. ${ }^{39}$ Recent experiments suggest that the barrier height strongly depends on the precise orientation of the $\mathrm{O}_{2}$ molecule. ${ }^{46}$ Obtaining a full six-dimensional potential energy surface, as required for dynamics simulations, is unfortunately challenging due to the considerable computational cost associated with the embedded $\mathrm{CW}$ treatment. Work along these lines is in progress.

To elucidate the origin of the observed energy barrier, we evaluate the charge and spin states of the approaching $\mathrm{O}_{2}$. We find an abrupt change in these states at the top of the barrier [see Figure 3c,d], suggesting charge transfer is suddenly initiated at a distance of $\sim 2.3 \AA$ from the surface and is responsible for the adsorption barrier. The lack of a barrier in conventional KS-DFT simulations is related to the lack of a derivative discontinuity with respect to the electron number in many conventional XC functionals. ${ }^{45}$ The absence of a derivative discontinuity introduces spurious long-range charge transfer between $\mathrm{O}_{2}$ and the aluminum surface, which in turn artificially removes the dissociation barrier observed in experiments and our embedding simulations.

\subsection{Hot-Electron-Mediated Dissociation of $\mathrm{H}_{2}$ on Gold}

A second example of the utility of DFET is treatment of local excited states in condensed matter. Earlier work demonstrated DFET's ability to treat excited states of adsorbed molecules and Kondo systems. ${ }^{20-22,25,26}$ Excited state chemistry is the next frontier. The example we consider is plasmon-induced catalysis, which offers the promise of efficiently driving chemical reactions at low temperature and under ambient conditions. ${ }^{47}$ The experiment proceeds as follows. One optically generates surface plasmons, that is, a collective oscillation of the electron cloud, in a metal nanoparticle. For noble-metal nanoparticles of suitable geometries, these excitations mostly decay into electron-hole pairs of (approximately) known energy, namely, the energy of the surface plasmon. ${ }^{48}$ Noble-metal nanoparticles thus offer efficient means to generate hot electrons with high throughput and low energy requirements at their surfaces. Nanoparticle properties such as size, geometry, and choice of metal readily allow tailoring the hot electron energy to match the antibonding state of a specific adsorbed molecule one wishes to dissociate. One could thus envision efficiently and selectively enhancing catalytic reactions by aiming for the energies of antibonding orbitals.

Understanding plasmon-mediated catalysis requires reliable theoretical methods to predict energies of specific antibonding orbitals of molecules near surfaces. Modeling of plasmoninduced hot electrons requires treating excited states. Although the linear-expansion delta self-consistent field extension of $\mathrm{DFT}^{49}$ has been used to approximate excited-state potential energy surfaces to model hot-electron adsorbate interactions, ${ }^{50}$ a more accurate treatment is beyond the scope of a groundstate theory like DFT. While excited states featuring substantial density rearrangement require state-specific embedding potentials, ${ }^{51}$ excitations localized within the embedded region allow for a simpler treatment. ${ }^{20-22,24-27,52}$ Due to the short screening lengths of metals, we choose the latter for metal nanoparticles. Describing the excited electron at the nanoparticle surface requires both treatment of exited states and consideration of the surface. Our embedding ansatz again furnishes the combination of a periodic DFT description of the metal
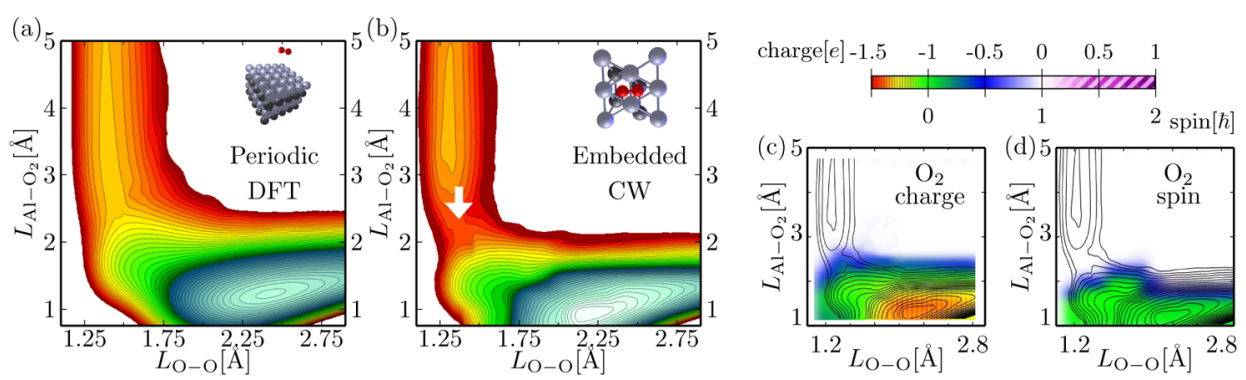

Figure 3. Potential energy surface (PES) of parallel $\mathrm{O}_{2}$ approaching $\mathrm{Al}(111)$ at the bridge site as a function of $\mathrm{O}_{2}$ bond length and surface distance calculated using (a) periodic DFT and (b) embedded CW theory on the cluster shown in Figure $2 .{ }^{31}$ The embedded CW PES features a barrier toward dissociation (see white arrow) of $\sim 600 \mathrm{meV}$, in excellent agreement with experiment. Contour line separation is $200 \mathrm{meV}$. (c) Charge and (d) spin state of $\mathrm{O}_{2}$ using embedded CW theory. See inset for color codes. Reproduced with permission from ref 31 . Copyright 2012 American Physical Society. 
surface with a CW treatment of excited electrons in the embedded cluster.

As a proof of principle, plasmons from Au nanoparticles were recently used to efficiently generate hot electrons that subsequently enabled $\mathrm{H}_{2}$ dissociation on $\mathrm{Au}$ nanoparticles [Figure 4]. ${ }^{32}$ Using a laser of suitable wavelength, plasmon
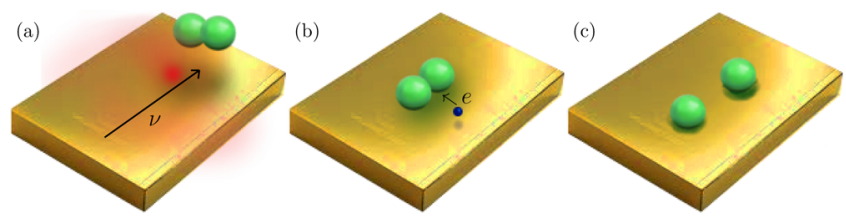

Figure 4. Schematic view of $\mathrm{H}_{2}$ dissociation on $\mathrm{Au}$ by plasmoninduced hot electron: (a) incoming photon, $\nu$ (red), generates (b) a hot electron, $e$ (blue), that jumps into the antibonding orbital of a physisorbed $\mathrm{H}_{2}$ molecule (green), (c) leading to dissociation.

resonances in gold nanoparticles embedded in a titania substrate were excited [Figure 5a]. Under an atmosphere of hydrogen $\left(\mathrm{H}_{2}\right)$ and deuterium $\left(\mathrm{D}_{2}\right)$, one measures the concentration of $\mathrm{HD}$ as a direct measure of dissociation and recombination of hydrogen molecules [Figure 5b]. Using our embedding ansatz, we calculated potential energy surfaces of ground and excited states. ${ }^{32,33}$ We found a large decrease in effective barrier height at specific excitation energies just below the plasmon resonance energy, corresponding to a Feshbach resonance of $\mathrm{H}_{2}^{-}$[compare Figure $5 \mathrm{~d}, \mathrm{f}$ ], offering a clear explanation of why dissociation is observed with the laser on: the embedded CW properly describes charge transfer excited states from the metal surface to the molecule; charge transfer weakens the bond by populating the antibonding molecular orbital; and the barrier in the excited state with the most charge transfer character (the sixth excited state) decreases dramatically to allow dissociation at low temperatures.

\section{POTENTIAL-FUNCTIONAL EMBEDDING THEORY: A SELF-CONSISTENT EMBEDDING FRAMEWORK}

The non-self-consistent embedding potential formulation outlined in section 2 does not consider changes in the environment due to the CW description of the cluster. This is an acceptable solution for cases where the screening length is extremely short (a few ångströms) such as metals. Here, charge transfer is screened on the length scale of the cluster size and therefore the remaining environment may be approximated as frozen. For semiconductors or semimetals, the screening length may be much larger. In such cases, charge transfer upon adsorption may change the surface electronic structure beyond the cluster length scale. The embedding potential thus needs to be updated using input from the CW description beyond the non-self-consistent DFET (section 2) approach.

To overcome the above difficulties, we have developed potential-functional embedding theory (PFET), ${ }^{30}$ where the basic underlying variable to optimize is the embedding potential, instead of electron densities. Such a formalism is based on the fact that the embedding potential $V_{\text {emb }}$ is a unique property of the total system for a given system partitioning. ${ }^{34}$

In PFET, the energy of the entire system (consisting of $\alpha=$ $1, \ldots, K$ subsystems) is given by

$$
E_{\text {tot }}=\sum_{\alpha} E_{\alpha}\left[\rho_{\alpha}, V_{\mathrm{emb}}\right]+E_{\mathrm{int}}\left[V_{\mathrm{emb}}, \rho_{1}, \rho_{2}, \ldots, \rho_{K}\right]
$$

where $E_{\alpha}\left[\rho_{\alpha}, V_{\mathrm{emb}}\right]$ is the subsystem energy functional that depends on subsystem electron densities $\rho_{\alpha}$ and $V_{\text {emb }}$. $E_{\text {int }}$ describes the interaction between subsystems. The $\left\{\rho_{\alpha}\right\}$ are obtained as the ground-state electron densities of the (a)
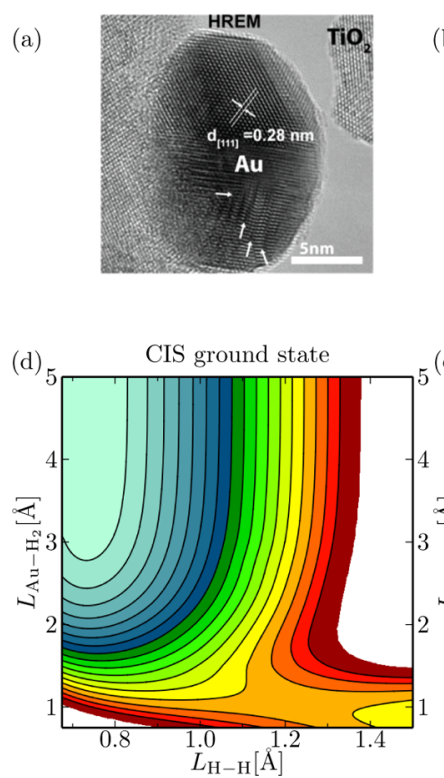

(b)
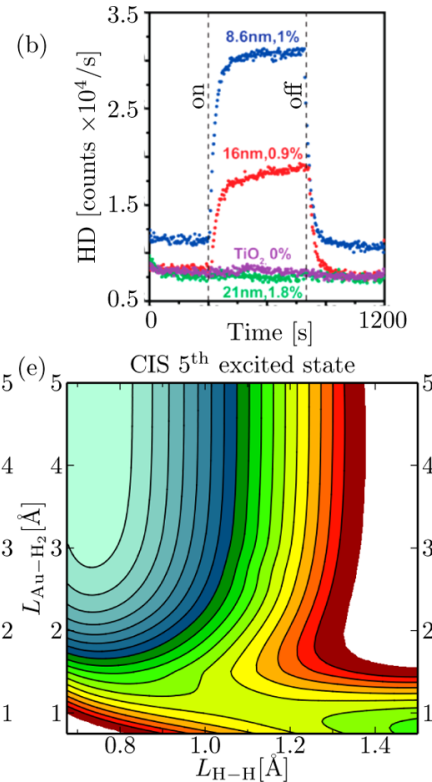

(c)
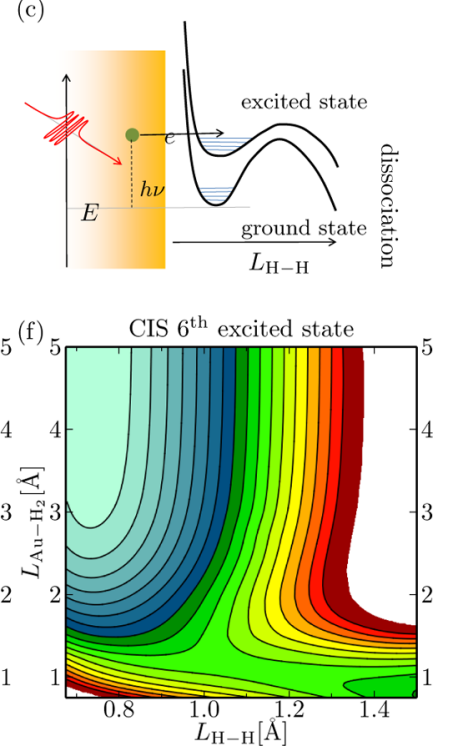

Figure 5. (a) High resolution transmission electron micrograph of a gold nanoparticle on a $\mathrm{TiO}_{2}$ substrate, used for demonstrating plasmonmediated catalysis. Interference fringes of $d=0.28 \AA$ indicative of $\mathrm{Au}(111)$ facets. (b) Measured $\mathrm{HD}$ ratio as a function of time for different nanoparticle diameters. Laser is turned on (see "on") at first dashed line $(t=300 \mathrm{~s})$, leading to a sudden substantial increase in HD count rate. As soon as laser is switched off again (see "off", $t=750 \mathrm{~s}$ ), $\mathrm{HD}$ count rate drops. Pristine $\mathrm{TiO}_{2}$ substrate shows no measurable $\mathrm{HD}$ count rate. (c) Schematic representation of dissociation by electronic excitation. (d-f) Embedded CW potential energy surface of parallel $\mathrm{H}_{2}$ approaching $\mathrm{Au}(111)$ in (d) ground, (e) fifth, and (f) sixth excited states of hydrogen adsorbed on $\mathrm{Au}(111){ }^{33}$ The height of the barrier substantially decreases. Reproduced with permission from ref 32. Copyright 2013 American Chemical Society. 
subsystems with $V_{\text {emb }}$ as an additional external potential. The number of electrons $N_{\alpha}$ in each subsystem is chosen such that the chemical potentials $\mu_{\alpha}$ in all subsystems are equal, $\mu_{\alpha}=$ $\mu \forall \alpha$, for a given $V_{\mathrm{emb}}$, providing the means for intersubsystem charge transfer. Thus, subsystem electron numbers implicitly depend on $V_{\text {emb }}$. The electron density of the total system, given as a superposition of the subsystem electron densities, is therefore a functional of $V_{\mathrm{emb}}$. Because of the HohenbergKohn theorems, the total system is uniquely determined by its ground state electron density. We therefore search for the $V_{\mathrm{emb}}$ that reproduces this unique electron density. In practice, we obtain $V_{\text {emb }}$ by minimizing the total energy with respect to $V_{\text {emb }}$, with subsystem electron numbers $\left\{N_{\alpha}\right\}$ fixed. A flowchart describing how to solve PFET using a nested loop is given in Figure 6. The inner loop optimizes $V_{\mathrm{emb}}$, and the outer loop optimizes $\left\{N_{\alpha}\right\}$.

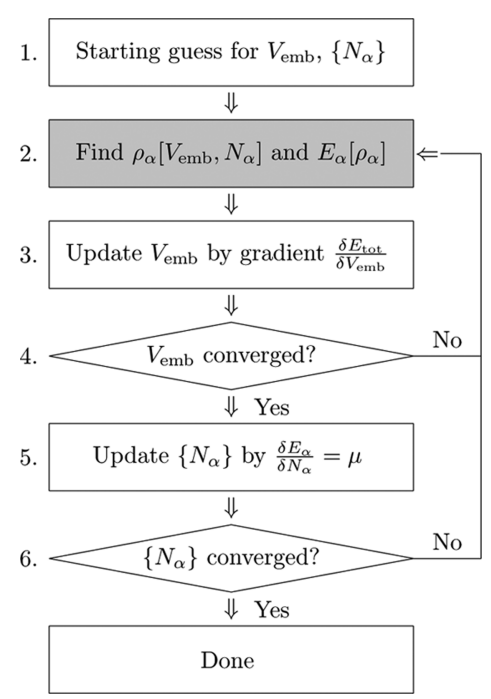

Figure 6. Flowchart for a PFET calculation. The shaded step can be executed independently for the different subsystems. ${ }^{30}$ Step 1 , an initial guess for $V_{\text {emb }}$ and the subsystem electron numbers $\left\{N_{\alpha}\right\}$ is generated. Step 2, subsystems are solved independently by taking $V_{\text {emb }}$ as an additional external potential. Subsystem electron densities and ground state energies are then obtained. Step 3, the gradient $\delta E_{\text {tot }} /$ $\delta V_{\text {emb }}$ is calculated according to ref 30 . Step 4, the convergence of $V_{\text {emb }}$ is tested. Step 5, If $V_{\mathrm{emb}}$ is converged, $\left\{N_{\alpha}\right\}$ are updated by equilibrating chemical potentials among subsystems. Step 6 , the convergence of $\left\{N_{\alpha}\right\}$ is tested. If the $\left\{N_{\alpha}\right\}$ are converged, the PFET calculation is finished. Otherwise we go back to step 1 to reoptimize $V_{\text {emb }}$ for this new set of $\left\{N_{\alpha}\right\}$.

To minimize the total energy with respect to $V_{\mathrm{emb}}$, one needs to compute the gradient $\delta E_{\text {tot }} / \delta V_{\text {emb }}$, which can be evaluated using an efficient finite difference scheme. ${ }^{30}$ For a subsystem not treated by KS-DFT, one needs to obtain the KS potential for its electron density by inverting the KS equations, in order to avoid using approximate KEDFs in the calculation of the KS kinetic energy component in $E_{\text {int. }}{ }^{8,53-55}$ Many ways to invert the KS equations exist, such as suggested by Wu and Yang ${ }^{56}$ and Zhao, Morrison, and Parr. ${ }^{57}$

In PFET, $V_{\mathrm{emb}}$ is the minimizer of the total energy. Thus, there is no longer an obvious connection between $\delta E_{\text {int }} / \delta \rho_{\alpha}$ and $V_{\text {emb }}$ [eq 2], as in DFET (section 2), where each subsystem electron density is a minimizer of the total energy. In PFET, any approximation to $E_{\text {int }}$ (such as evaluating $E_{\text {int }}$ within KSDFT instead of using the same CW method(s) used for each subsystem) will cause subsystem densities to no longer globally minimize the total energy.

Changing the variable from electron density to embedding potential, $V_{\mathrm{emb}}$, brings many new exciting possibilities. It becomes straightforward to enforce all subsystems to share the same embedding potential, required for the uniqueness of $V_{\text {emb. }}{ }^{30}$ Different subsystems can be treated with different quantum mechanics methods: $E_{\alpha}\left[V_{\mathrm{emb}}, N_{\alpha}\right]$ can be evaluated with a quantum mechanics method appropriate to the physics of that subsystem. The interaction term $E_{\text {int }}$ also can be approximated to reduce computational cost while preserving the correct physics. ${ }^{30}$ Therefore, PFET opens the door to seamless, self-consistent, first-principles embedding simulations.

Technically, PFET is a powerful framework that can be used in conjunction with different computer programs. Each subsystem can be calculated using any level of theory as long as a local, external potential can be input and the ground-state energy and electron density are output. The numerically expensive calculations are performed independently for each subsystem (see shaded box in Figure 6) and thus can be efficiently parallelized. Fractional electron numbers in subsystems can be derived from KS-DFT with KS orbitals fractionally occupied, or from the ensemble DFT approach. ${ }^{45}$ Since it is the total electron density that determines the entire system, we can treat fractional electron numbers in subsystems in whatever way is convenient. We have benchmarked PFET within pure KS-DFT for molecules and materials. ${ }^{30} \mathrm{We}$ find excellent agreement with KS-DFT benchmarks in all cases. We are currently working on practical tests for CW-in-DFT embedding using PFET, as well as a recently developed timedependent PFET. ${ }^{58}$

\section{CONCLUSIONS}

A number of fundamental as well as technologically important phenomena involve processes and states of matter poorly described by the quantum mechanics workhorse known as density functional theory (DFT). Refined electronic structure methods are needed to understand basic mechanisms of, for example, charge transfer that are key to such contemporary challenges as efficient electrochemical energy conversion. The latter is critical to facilitating clean, efficient electricity generation via fuel cells and likewise generating fuels from (photo)-electrocatalysis. Conventional DFT approximations fail to treat charge transfer excitations correctly due to $\mathrm{XC}$ functional limitations. In this Account, we reviewed recent advances in embedded correlated wavefunction (CW) theory and how this theory has been used to understand (photo)electrochemical reactions at metal surfaces. Embedded CW theory treats charge transfer and excited states accurately by properly including exact electron exchange and correlation in a region of interest, while the extended metal background is described via periodic DFT, encapsulated in an embedding potential. We saw that embedded CW theory can properly describe the first step of the oxygen reduction reaction that occurs at fuel cell cathodes, namely, charge-transfer-induced $\mathrm{O}_{2}$ dissociation at the metal surface. By contrast, conventional DFT completely misses quantitative and qualitative features of the reaction. We also saw that an unusual form of (photo)electrocatalysis can be captured by this theory, namely, plasmon-induced hot electron dissociation of molecules on gold nanoparticles, by revealing that charge transfer excited states weaken bonds in impinging molecules so they can more easily dissociate, even on inert substrates such as gold. 
Thus, density-based embedding of CWs can accurately treat many interesting phenomena in which a single quantum mechanical method proves insufficient to tackle the full problem complexity. The more general potential-functional embedding, which allows for charge equilibration across subsystems to achieve full self-consistency, has only been tested at the pure DFT level thus far. Further developments aim at self-consistently evaluating the embedding potential to include the back-action of high-level CW methods on the environment or multiple subsystems, as well as extending fully self-consistent embedded CW theory to the time-dependent domain.

\section{AUTHOR INFORMATION}

\section{Corresponding Author}

*E-mail: eac@princeton.edu.

\section{Funding}

F.L. acknowledges support by the SFB-041 ViCoM, and E.A.C. is grateful to the U.S. National Science Foundation for support of this research.

Notes

The authors declare no competing financial interest.

\section{Biographies}

Dr. Florian Libisch graduated from the Vienna University of Technology with a Ph.D. in theoretical physics in 2009. He was a postdoctoral researcher at Princeton University for two years and currently holds a faculty position in Vienna.

Dr. Chen Huang is a postdoctoral research associate in the Theoretical Division at Los Alamos National Laboratory. He received his Ph.D. in Physics from Princeton University in 2011 and his B.Sc. from Tsinghua University in Beijing, China.

Prof. Emily A. Carter is the Founding Director of the Andlinger Center for Energy and the Environment at Princeton University and the Gerhard R. Andlinger Professor in Energy and the Environment, as well as Professor of Mechanical and Aerospace Engineering and Applied and Computational Mathematics. She was educated at UC Berkeley (B.S. Chemistry), Caltech (Ph.D. Physical Chemistry), and the University of Colorado, Boulder (postdoctoral research).

\section{REFERENCES}

(1) Shavitt, I.; Bartlett, R. J. Many-Body Methods in Chemistry and Physics: MBPT and Coupled-Cluster Theory; Cambridge University Press: Cambridge, U.K., 2009.

(2) Krisiloff, D.; Dieterich, J.; Libisch, F.; Carter, E. A. Numerical Challenges in a Cholesky-decomposed Local Correlation Quantum Chemistry Framework. In Mathematical and Computational Modeling: With Applications in Engineering and the Natural and Social Sciences, Melnick, R., Ed.; Wiley: New York, 2014, ISBN: 1118853989.

(3) Schütz, M.; Werner, H.-J. Low-order scaling local electron correlation methods. IV. Linear scaling local coupled-cluster (LCCSD). J. Chem. Phys. 2001, 114, 661-681.

(4) Maslen, P. E.; Head-Gordon, M. Non-iterative local second order Möller-Plesset theory. Chem. Phys. Lett. 1998, 283, 102-108.

(5) Pisani, C.; Busso, M.; Capecchi, G.; Casassa, S.; Dovesi, R.; Maschio, L.; Zicovich-Wilson, C.; Schütz, M. Local-MP2 electron correlation method for nonconducting crystals. J. Chem. Phys. 2005, 122, No. 094113.

(6) Grüneis, A.; Booth, G. H.; Marsman, M.; Spencer, J.; Alavi, A.; Kresse, G. Natural orbitals for wavefunction based correlated calculations using a plane wave basis set. J. Chem. Theory Comput. 2011, 7, 2780-2785.
(7) Booth, G. H.; Grüneis, A.; Kresse, G.; Alavi, A. Towards an exact description of electronic wavefunctions in real solids. Nature 2013, 493, 365-370.

(8) Huang, P.; Carter, E. A. Advances in correlated electronic structure methods for solids, surfaces, and nanostructures. Annu. Rev. Phys. Chem. 2008, 59, 261-290.

(9) Dick, B. G., Jr.; Overhauser, A. W. Theory of the dielectric constants of alkali halide crystals. Phys. Rev. 1958, 112, 90-103.

(10) Karplus, M.; Levitt, M.; Warshel, A. Nobel Prizes 2013. Angew. Chem., Int. Ed. 2013, 52, 11972.

(11) Vreven, T.; Morokuma, K. Chapter three: Hybrid methods: ONIOM(QM:MM) and QM/MM. Ann. Rep. Comput. Chem. 2006, 2, $35-51$.

(12) Manby, F. R.; Stella, M.; Goodpaster, J. D.; Miller, T. F. A simple, exact density-functional-theory embedding scheme. J. Chem. Theory Comput. 2012, 8, 2564-2568.

(13) Goodpaster, J. D.; Barnes, T. A.; Manby, F. R.; Miller, T. F. Density functional theory embedding for correlated wavefunctions: Improved methods for open-shell systems and transition metal complexes. J. Chem. Phys. 2012, 137, No. 224113.

(14) Knizia, G.; Chan, G. Density matrix embedding: A simple alternative to dynamical mean-field theory. Phys. Rev. Lett. 2012, 109, No. 186404.

(15) Inglesfield, J. E. A method of embedding. J. Phys. C 1981, 14, 3795-3806.

(16) Cortona, P. Self-Consistently Determined Properties of Solids without Band-Structure Calculations. Phys. Rev. B 1991, 44, 84548458.

(17) Wesolowski, T. A.; Warshel, A. Frozen density functional approach for ab-initio calculations of solvated molecules. J. Phys. Chem. 1993, 97, 8050-8053.

(18) Govind, N.; Wang, Y. A.; da Silva, A. J. R.; Carter, E. A. Accurate ab initio energetics of extended systems via explicit correlation embedded in a density functional environment. Chem. Phys. Lett. 1998, 295, 129-134.

(19) Govind, N.; Wang, Y. A.; Carter, E. A. Electronic-structure calculations by first-principles density-based embedding of explicitly correlated systems. J. Chem. Phys. 1999, 110, 7677-7688.

(20) Klüner, T.; Govind, N.; Wang, Y. A.; Carter, E. A. Prediction of electronic excited states of adsorbates on metal surfaces from first principles. Phys. Rev. Lett. 2001, 86, 5954-5957.

(21) Klüner, T.; Govind, N.; Wang, Y. A.; Carter, E. A. Periodic density functional embedding theory for complete active space selfconsistent field and configuration interaction calculations: Ground and excited states. J. Chem. Phys. 2002, 116, 42-54.

(22) Klüner, T.; Govind, N.; Wang, Y. A.; Carter, E. A. Reply to the comment on 'Prediction of Electronic Excited States of Adsorbates on Metal Surfaces from First Principles'. Phys. Rev. Lett. 2002, 88, No. 209702.

(23) Sharifzadeh, S.; Huang, P.; Carter, E. A. Embedded configuration interaction description of $\mathrm{CO}$ on $\mathrm{Cu}(111)$ : Resolution of the site preference conundrum. J. Phys. Chem. C 2008, 112, 46494657.

(24) Huang, P.; Carter, E. A. Self-consistent embedding theory for locally correlated configuration interaction wavefunctions in condensed matter. J. Chem. Phys. 2006, 125, No. 084102.

(25) Huang, P.; Carter, E. A. Local electronic structure around a single Kondo impurity. Nano Lett. 2006, 6, 1146-1150.

(26) Huang, P.; Carter, E. A. Ab initio explanation of tunneling line shapes for the Kondo impurity state. Nano Lett. 2008, 8, 1265-1269.

(27) Sharifzadeh, S.; Huang, P.; Carter, E. A. Origin of tunneling lineshape trends for Kondo states of Co adatoms on coinage metal surfaces. J. Phys.: Condens. Matter 2009, 21, No. 355501.

(28) Sharifzadeh, S.; Huang, P.; Carter, E. A. All-electron embedded correlated wavefunction theory for condensed matter electronic structure. Chem. Phys. Lett. 2009, 470, 347-352.

(29) Huang, C.; Pavone, M.; Carter, E. A. Quantum mechanical embedding theory based on a unique embedding potential. J. Chem. Phys. 2011, 134, No. 154110. 
(30) Huang, C.; Carter, E. A. Potential-functional embedding theory for molecules and materials. J. Chem. Phys. 2011, 135, No. 194104.

(31) Libisch, F.; Huang, C.; Liao, P.; Pavone, M.; Carter, E. A. Origin of the energy barrier to chemical reactions of $\mathrm{O}_{2}$ on $\mathrm{Al}(111)$ : Evidence for charge transfer not spin selection. Phys. Rev. Lett. 2012, 109, No. 198303.

(32) Mukherjee, S.; Libisch, F.; Large, N.; Neumann, O.; Brown, L. V.; Cheng, J.; Lassiter, B.; Carter, E. A.; Nordlander, P.; Halas, N. J. Hot electrons do the impossible: Plasmon-induced dissociation of $\mathrm{H}_{2}$ on Au. Nano Lett. 2013, 13, 240-247.

(33) Libisch, F.; Cheng, J.; Carter, E. A. Electron-transfer-induced dissociation of $\mathrm{H} 2$ on gold nanoparticles: Excited-state potential energy surfaces via embedded correlated wave-function theory. Z. Phys. Chem. 2013, 227, 1455-1466.

(34) Cohen, M. H.; Wasserman, A. On hardness and electronegativity equalization in chemical reactivity theory. J. Stat. Phys. 2006, $125,1121-1139$.

(35) Cohen, M. H.; Wasserman, A. On the foundations of chemical reactivity theory. J. Phys. Chem. A 2007, 111, 2229-2242.

(36) Elliott, P.; Burke, K.; Cohen, M. H.; Wasserman, A. Partition density functional theory. Phys. Rev. A 2010, 82, No. 024501.

(37) Kroes, G.-J. Frontiers in surface scattering simulations. Science 2008, 321, 794-797.

(38) Zambelli, T.; Barth, J. V.; Wintterlin, J.; Ertl, G. Complex pathways in dissociative adsorption: $\mathrm{O} 2$ on $\mathrm{Pt}(111)$. Nature 1997, 390, 495-497.

(39) Österlund, L.; Zorić, I.; Kasemo, K. Dissociative sticking of $\mathrm{O}_{2}$ on $\mathrm{Al}(111)$. Phys. Rev. B 1997, 55, 15452-15455.

(40) Komrowski, A. J.; Sexton, J. Z.; Kummel, A. C.; Binetti, M.; Weiße, O.; Hasselbrink, E. Oxygen abstraction from dioxygen on the $\mathrm{Al}(111)$ surface. Phys. Rev. Lett. 2001, 87, No. 246103.

(41) Sasaki, T.; Ohno, T. Dissociation process of $\mathrm{O}_{2}$ on the $\mathrm{Al}(111)$ surface. Surf. Sci. 1999, 433, 172-175.

(42) Carbogno, C.; Behler, J.; Groß, A.; Reuter, K. Fingerprints for spin-selection rules in the interaction dynamics of $\mathrm{O}_{2}$ at $\mathrm{Al}(111)$. Phys. Rev. Lett. 2008, 101, No. 096104.

(43) Carbogno, C.; Behler, J.; Reuter, K.; Groß, A. Signatures of nonadiabatic $\mathrm{O}_{2}$ dissociation at $\mathrm{Al}(111)$ : First-principles fewestswitches study. Phys. Rev. Lett. 2008, 101, No. 096104.

(44) Mosch, C.; Koukounas, C.; Bacalis, N.; Metropoulos, A.; Gross, A.; Mavridis, A. Interaction of dioxygen with $\mathrm{Al}$ clusters and $\mathrm{Al}(111)$ : A comparative theoretical study. J. Phys. Chem. C 2008, 112, 69246932.

(45) Perdew, J. P.; Levy, M.; Balduz, J. L. Density-functional theory for fractional particle number: Derivative discontinuities of the energy. Phys. Rev. Lett. 1982, 49, 1691-1693.

(46) Kurahashi, M.; Yamauchi, Y. Steric effect in $\mathrm{O}_{2}$ sticking on $\mathrm{Al}$ (111): Preference for parallel geometry. Phys. Rev. Lett. 2013, 110, No. 246102.

(47) Linic, S.; Christopher, P.; Ingram, D. B. Plasmonic-metal nanostructures for efficient conversion of solar to chemical energy. Nat. Mater. 2011, 10, 911-921.

(48) Fang, Z.; Wang, Y.; Liu, Z.; Schlather, A.; Ajayan, P. M.; Koppens, F. H. L.; Nordlander, P.; Halas, N. J. Plasmon-induced doping of graphene. ACS Nano 2012, 6, 10222-10228.

(49) Gavnholt, J.; Olsen, T.; Engelund, M.; Schiøtz, J. $\Delta$ Selfconsistent field method to obtain potential energy surfaces of excited molecules on surfaces. Phys. Rev. B 2008, 78, No. 075441.

(50) Christopher, P.; Xin, H.; Marimuthu, A.; Linic, S. Singular characteristics and unique chemical bond activation mechanisms of photocatalytic reactions on plasmonic nanostructures. Nat. Mater. 2012, 11, 1044-1050.

(51) Daday, C.; König, C.; Valsson, O.; Neugebauer, J.; Filippi, C. State-specific embedding potentials for excitation-energy calculations. J. Chem. Theory Comput. 2013, 9, 2355-2367.

(52) Khait, Y. G.; Hoffmann, M. R. Embedding theory for excited states. J. Chem. Phys. 2010, 133, No. 044107.

(53) Fux, S.; Jacob, R. C.; Neugebauer, J.; Visscher, L.; Reiher, M. Accurate frozen-density embedding potentials as a first step towards a subsystem description of covalent bonds. J. Chem. Phys. 2010, 132, No. 164101.

(54) Nafziger, J.; Wu, Q.; Wasserman, A. Molecular binding energies from partition density functional theory. J. Chem. Phys. 2011, 135, No. 234101.

(55) Goodpaster, J. D.; Ananth, N.; Manby, F. R.; Miller, T. F. Exact nonadditive kinetic potentials for embedded density functional theory. J. Chem. Phys. 2010, 133, No. 084103.

(56) Wu, Q.; Yang, W. A direct optimization method for calculating density functionals and exchange-correlation potentials from electron densities. J. Chem. Phys. 2003, 118, 2498-2509.

(57) Zhao, Q.; Morrison, R. C.; Parr, R. G. From electron densities to Kohn-Sham kinetic energies, orbital energies, exchange-correlation potentials, and exchange-correlation energies. Phys. Rev. A 1994, 50, $2138-2142$.

(58) Huang, C.; Libisch, F.; Peng, Q.; Carter, E. A. Time-dependent potential-functional embedding theory. J. Chem. Phys. 2014, 140, No. 124113. 